\title{
Transverse magnetization and torque in asymmetrical mesoscopic superconductors
}

\author{
Antonio R. de C. Romaguera, ${ }^{1,2, *}$ Mauro M. Doria, ${ }^{2,1}$ and F. M. Peeters ${ }^{2}$ \\ ${ }^{1}$ Instituto de Física, Universidade Federal do Rio de Janeiro, Caixa Postale 68.528, 21941-972 Rio de Janeiro, Brazil \\ ${ }^{2}$ Departement Fysica, Universiteit Antwerpen, Groenenborgerlaan 171, B-2020 Antwerpen, Belgium
}

(Received 15 May 2007; published 13 July 2007)

\begin{abstract}
We show that asymmetrical mesoscopic superconductors bring new insight into vortex physics, where we found the remarkable coexistence of long and short vortices. We study an asymmetrical mesoscopic sphere that lacks one of its quadrants, and obtain its three-dimensional vortex patterns by solving the Ginzburg-Landau theory. We find that the vortex patterns are asymmetric, and their effects are clearly visible and detectable in the transverse magnetization and torque.
\end{abstract}

DOI: 10.1103/PhysRevB.76.020505

PACS number(s): 74.78.Na, 74.25.Ha, 74.20.-z, 74.62.Dh

The small volume to surface ratio of mesoscopic superconductors makes their properties distinct from those of bulk materials. ${ }^{1}$ For instance, in the absence of pinning and anisotropy, mesoscopic superconductors have an intrinsic magnetic hysteresis, whereas bulk samples do not. Mesoscopic superconductors are intrinsically metastable, since they can be in excited vortex states due to an energetic barrier slowing the decay to the ground state., ${ }^{2,3}$ Several experimental techniques have been developed to detect mesoscopic vortex states $^{4-6}$ and to reveal their new and interesting physical properties. ${ }^{7,8}$ Here we obtain the transverse magnetization and torque that result from the asymmetrical coexistence of vortices with different lengths in mesoscopic superconductors. The general aspects of our results apply to irregularly shaped grains, and, consequently, can also be relevant to the understanding of inhomogeneous bulk samples with granular structure. ${ }^{9-11}$

In this paper, we obtain and compare the vortex patterns of two similar but distinct mesoscopic systems, namely, a full sphere with radius $R=6.0 \xi$ and an asymmetrical sphere, with the same radius but lacking one of its quadrants. Hereafter we call it the minus-quarter sphere, and we present here its transverse magnetization and torque.

There are many reasons for the magnetic moment of a sample with volume $V, \boldsymbol{\mu}=V \boldsymbol{M}$, not to be aligned along the applied magnetic field $\boldsymbol{H}$. A direct measurement ${ }^{12-14}$ of the torque

$$
\boldsymbol{\tau}=\boldsymbol{\mu} \times \boldsymbol{H}=H \mu \sin \theta \hat{\boldsymbol{e}}_{\tau},
$$

brings insight about these distinct contributions. ${ }^{15,16}$ Here $\theta$ is the angle between $\boldsymbol{H}$ and $\boldsymbol{M}$. For example, vortices pinned by pointlike normal inclusions (defects) are major contributors to torque, due to their inability to follow a rotating applied field. This contribution is usually avoided as one seeks out true information about intrinsic properties of the superconducting state. The sample's shape and size also contribute, and provide the basis for very high-precision magnetization measurements using torque. ${ }^{14}$ Basically, measurement of the torque relies on the thin film limit, where vortices remain straight and in one oriented direction inside the sample upon field rotation, resulting in a magnetic sign always perpendicular to the major surfaces. The general misalignment between the applied field and the sample's major axis produces a torque, even for a regularly shaped superconductor. Hence, to obtain a torque it suffices that the circulating supercurrent yields a magnetic field response not oriented along the applied field that gave rise to it. Asymmetry leads to such an effect, and we show here that it provides a useful tool to probe the physics of mesoscopic superconductors.

In this paper we provide a study of a mesoscopic superconductor with a transverse $\left(M_{x}\right)$ magnetization, thanks to the use of a truly three-dimensional approach. ${ }^{17}$ The effects found in the transverse magnetization and in the torque are within reach of present experimental detection. ${ }^{14}$ For instance, a coherence length of $\xi \simeq 100 \mathrm{~nm}$ leads to $H_{c 2}$ $=\Phi_{0} / 2 \pi \xi^{2} \simeq 300 \mathrm{G}^{18}$ If the penetration length is comparable to the sphere's radius $R=0.6 \mu \mathrm{m}$, then the transverse magnetization is approximately ten times smaller, $M_{x}$ $\simeq 10^{-3} H_{c 2} \simeq 0.3 \mathrm{G}$, than the longitudinal one, $M_{z} \simeq 10^{-2} H_{c 2}$ $\simeq 3 \mathrm{G}$. The magnetic moment of this sphere is $\mu \sim M_{z} V$ $\simeq 7.0 \times 10^{-12} \mathrm{emu} \quad\left(V=72 \pi \xi^{3} \simeq 7.63 \times 10^{-13} \mathrm{~cm}^{3}\right.$ for the minus-quarter sphere). The torque $\tau_{y}=-M_{x} H_{z} V \simeq 10^{-3} H_{c 2}^{2} V$ becomes $\tau_{y}=4.0 \times 10^{-12} \mathrm{erg}$.

Figure 1 summarizes the aspects introduced by asymmetry, showing the Cooper pair density $|\psi(\mathbf{r})|^{2}$ obtained from our numerical simulations for selected values of the applied field. States with at most three vortices were selected to help to understand the basic effects caused by asymmetry. For all cases, the magnetic field is oriented along the direction from bottom to top. In those mesoscopic systems, vortices are naturally curved ${ }^{19}$ because the entrance and exit of the vortex lines must be perpendicular to the surface, ${ }^{20}$ a fact that causes a closely packed configuration near the equatorial region, as previously found. ${ }^{17}$

Figure 1(d) best represents the effects of the asymmetry, where the position of the short vortex, very close to the center of the minus-quarter sphere, is determined by (i) the spherical shell pushing the vortex near the central axis where the shielding current offers maximal stability ${ }^{21}$ and (ii) the flat surface of the missing quadrant where the vortex length is half of that for a full sphere. As a consequence, the vortex is displaced from the central axis, breaking the rotational symmetry in the northern hemisphere and resulting in transverse magnetization and torque. The second vortex has the same destiny as the first one; it is also a short vortex that gets as close as possible to the central axis [Fig. 1(e)]. The situation changes for the third vortex, possibly because $R=6.0 \xi$ is just too small to accommodate it in the quadrant, forcing 
(g)
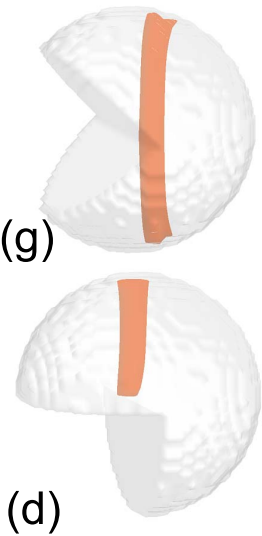

(d)

(a)

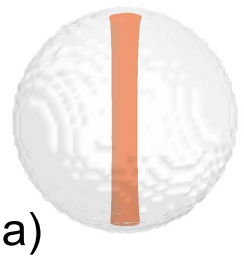

(b)

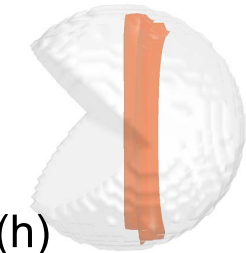

(h)

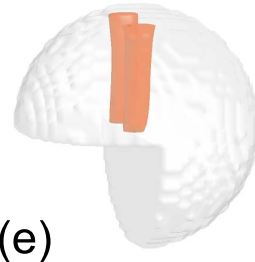

(f)

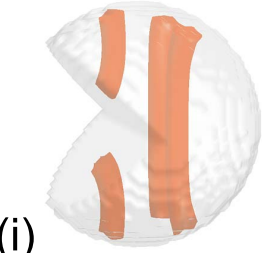

(i)

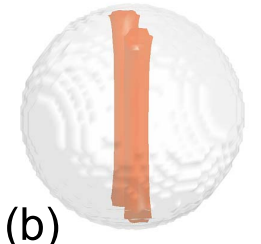

(c)
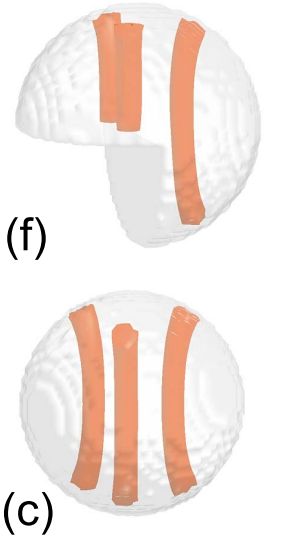

FIG. 1. (Color online) Three-dimensional isodensity plots taken at $20 \%$ of the maximum density $|\psi|^{2}$. Each isocontour is a single surface made of the vortices and the superconductor surface. The magnetic field is directed from bottom to top with $H / H_{c 2}=0.16$ (a), 0.29 (b), 0.35 (c), 0.27 (d), 0.39 (e), 0.49 (f), 0.35 (g), 0.48 (h), and 0.54 (i). The figures correspond to the full sphere (a)-(c) and to the minus-quarter sphere, positioned in two ways, (d)-(f) and (g)-(i), with respect to the field.

its nucleation in the full hemisphere, where it becomes the first long vortex. Asymmetry brings peculiar, not always intuitive, features. For instance, the rotation of the minusquarter sphere by $45^{\circ}$ leads to new vortex configurations not accessible in the case of the previous alignment. Now the long vortices nucleate first and short vortices enter in pairs afterwards, as shown in Figs. 1(g)-1(i), with increasing field. In summary, asymmetry creates new situations that can bring further information about vortex patterns in mesoscopic superconductors. They are detectable in the transverse magnetization and the torque, providing information about vortex patterns in such systems.

The Ginzburg-Landau theory, derived near the critical temperature, is applicable at much lower temperatures for mesoscopic superconductors, where it was found to give a fair description of the magnetic properties. ${ }^{4,18} \mathrm{We}$ solve the full three-dimensional Ginzbug-Landau theory for the asymmetrical superconductor by the numerical procedure described in Refs. 17 and 22, which minimizes the free energy with the boundary conditions included. In this approach the superconductor shape is characterized by a steplike function in the free energy ( 1 inside the superconductor and 0 outside). Here we also include an intermediate step inside the superconductor $(0.8$ within a layer of thickness $0.5 \xi)$ to stabilize the states for fields near $H_{c 3}$, according to Ref. 17 . The present results are obtained under the approximation that the applied field uniformly permeates the mesoscopic superconductor, so that the magnetic shielding is safely ignored. A discretized gauge invariant version of the Ginzburg-Landau

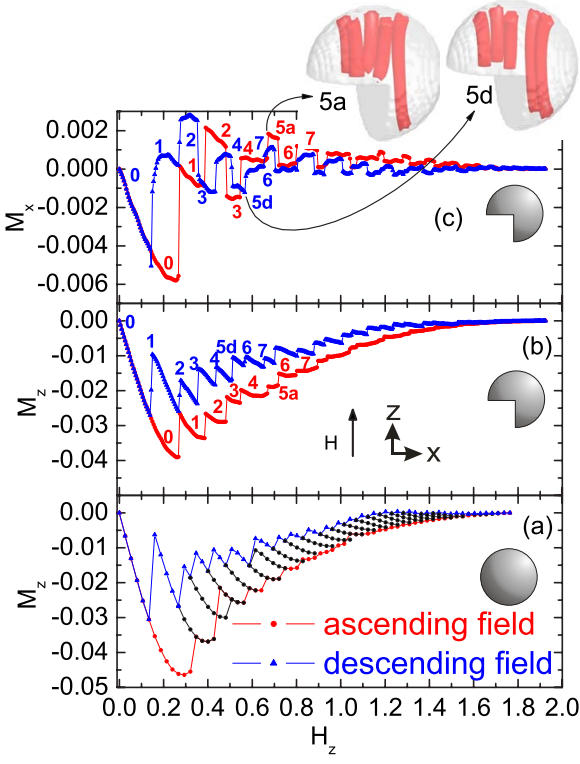

FIG. 2. (Color online) Magnetization for a full field cycle (a) for the full and (b), (c) for the minus-quarter sphere. The ascending (red) and descending (blue) branches are labeled for the first nine vortex states. Selected isoplots obtained in ascending and descending fields show the degeneracy of state 5 . The full nonintersecting magnetization branches are shown just for the full sphere.

free energy is minimized in a cubic mesh with $46 \times 46 \times 46$ points, ${ }^{17}$ and the magnetization is directly determined from $\boldsymbol{M}=$ const $\times(1 / 2 c) \int \boldsymbol{r} \times \boldsymbol{J} d v / V$, where $\boldsymbol{J}$ is the supercurrent in the mesoscopic superconductor volume, and the constant is a free parameter associated with the demagnetization tensor $^{23,24} \boldsymbol{D}$, and thus obtained in our numerical simulations from the zero-vortex branch (Meissner phase) at small $H$ : $\boldsymbol{H}+4 \pi \boldsymbol{D} \cdot \boldsymbol{M}=0$. We apply the inverted relations $M_{x}$ $=\left(-D_{z z} H_{x}+D_{x z} H_{z}\right) /$ det and $M_{z}=\left(D_{z x} H_{x}-D_{x x} H_{z}\right) /$ det with $\operatorname{det}=D_{z z} D_{x x}-D_{x z} D_{z x}$ in our numerical analysis. We find that for the full sphere the demagnetization tensor reduces to a single scalar $D$, as expected, which is adjusted to the wellknown value 23,24 of $D=1 / 3$, and for the minus-quarter sphere $D_{x x}=D_{z z}, D_{x z}=D_{z x}$, and also that $D_{z z} / D=1.20$ and $D_{z x} / D=-0.184$. In the rest of the paper we use the Gaussian system of units and express both the magnetization and the applied field in terms of $H_{c 2}$, the bulk upper critical field, and the torque per volume in units of $H_{c 2}^{2}$.

Figure 2 shows the magnetization of the full and the minus-quarter spheres. We use the notation long+short $\rightarrow$ (long, short) to express the minus-quarter sphere states; the first nine, labeled in Figs. 2(b) and 2(c), are given by 0 $\rightarrow(0,0), \quad 1 \rightarrow(0,1), 2 \rightarrow(0,2), 3 \rightarrow(1,2), 4 \rightarrow(1,3), 5 d$ $\rightarrow(1,4), 5 a \rightarrow(2,3), 6 \rightarrow(2,4)$, and $7 \rightarrow(2,5)$. Notice that there are two 5 vortex states, $5 a$ and $5 d$, the first being the lower in energy, and therefore reached by the ascending field. These two states can coexist under the same field, although Fig. 2 does not show the corresponding prolongation of the $5 a$ and $5 d$ curves. This degeneracy is a new and interesting aspect caused by asymmetry, which shows that the total number of vortices cannot be used as a label to identify a single magnetization branch. We found 24 states for the full 


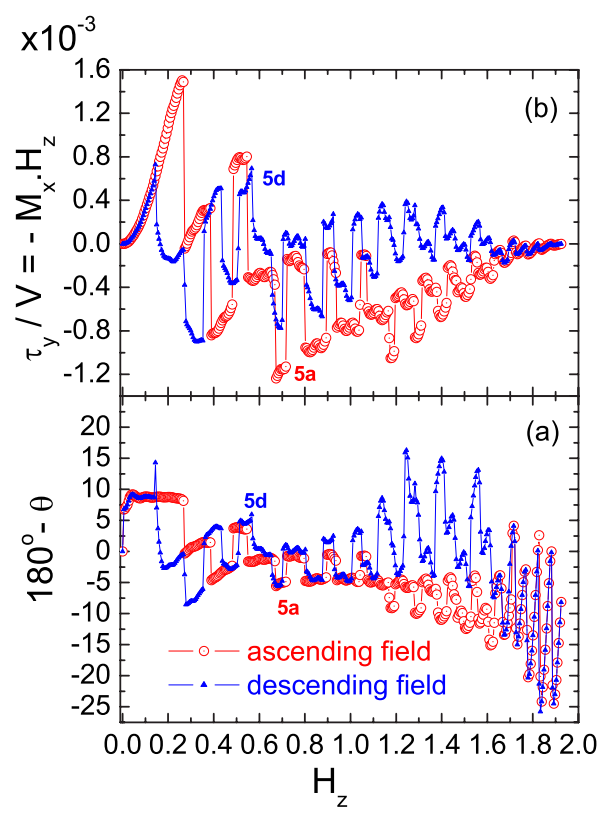

FIG. 3. (Color online) Ascending (red) and descending (blue) torque branches (a) and the corresponding misalignment angle (b).

sphere and at least 26 for the minus-quarter sphere. The higher vortex states, which exist near the normal state, become increasingly difficult to identify, and so require more precision than considered here. The magnetization curves were obtained in a full cycle field sweep that starts at zero, reaches the maximum possible value $\left(H_{c 3}\right)$ (ascending branch, red color), and finally is lowered back to zero (descending branch, blue color). Figures 2(a) and 2(b) show the magnetization component along the field, $M_{z}$, with the expected feature of rounded branches for the ascending field and a sawtooth structure for the descending field. This behavior has been extensively discussed both theoretically ${ }^{25-27}$ and experimentally. ${ }^{4}$ The full magnetization branch associated with a distinct vortex configuratrion is displayed here only for the full sphere [Fig. 2(a)]. Its construction requires numerous sweeps, starting at intermediate applied field values. Notice the distinct features of $M_{x}$ in comparison to $M_{z}$. The ascending and descending branches are entangled, and have an underlying structure that approximately breaks up into distinct families, associated with odd and even numbers of vortices. These branches display a wiggly structure due to vortex repositioning, which causes abrupt changes in vortex length, and so in energy and current flow.

The vortex entrance or exit can be detected through the transverse magnetization, whose sign changes accordingly, as seen in Fig. 2(c). Mesoscopic superconductors are known to be able to exhibit a paramagnetic signal when in a metastable vortex state, ${ }^{28,29}$ but this is not the case for the sphere, as the dominant component $\left(M_{z}\right)$ remains steadily oriented opposite to the applied field. However, a change of sign in $M_{x}$ implies that the torque flips direction, as shown in Fig. 3. Moreover, the torque is proportional to the applied field, and it becomes naturally enhanced in the regime between $H_{c 2}$ and $H_{c 3}$, in contrast to the magnetization, which becomes small in this region. The magnetization angle $180^{\circ}-\theta$ shows

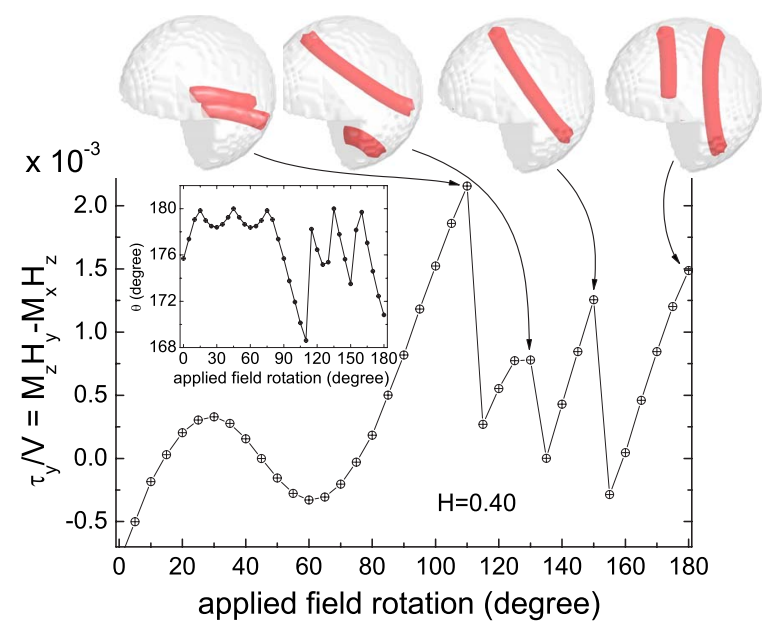

FIG. 4. (Color online) Torque upon rotation of the fixed applied field $(H=0.40)$ in $5^{\circ}$ increments. The inset shows the angle between $\boldsymbol{M}$ and $\boldsymbol{H}$ versus the applied field angle. Selected isoplot configurations for $110^{\circ}, 130^{\circ}, 150^{\circ}$, and $180^{\circ}$ are also displayed here.

that the misalignment with the applied field is about $10^{\circ}$ for small fields, and it oscillates above $H_{c 2}$. Thus, torque measurement can be very helpful in the detection of high vortex states, where the onset of a vortex configuration causes an abrupt change of sign. As an example, Fig. 4 shows the torque for a rotating field of intensity $H=0.4$. The selected initial configuration for $0^{\circ}$ is the ground state for this field, made of two short vortices [see Fig. 1(e)]. As the field rotates by angular increments of $5^{\circ}$, the two short vortices follow along, and this configuration remains stable until $110^{\circ}$, even though during this process the torque flips sign four times. The torque suffers an abrupt drop at $115^{\circ}$, signaling the entrance of a new vortex pattern. One of the short vortices is replaced by a long vortex, and the remaining short vortex moves to the center of the minus-quarter sphere. This long and short vortex configuration, i.e., $(1,1)$, lasts until the next torque transition at $130^{\circ}$, where a single long vortex becomes the only accessible configuration (from $135^{\circ}$ until $150^{\circ}$ ). Thus, the Fig. 1(g) state is reached in this way. The last abrupt drop brings back the $(1,1)$ configuration from $155^{\circ}$ to $180^{\circ}$. The final state does not coincide with the initial one, and is not included as one of the available stable configurations listed in Fig. 1. Thus it is a very highly excited state much above the ground state. Nevertheless, it became accessible by virtue of the torque.

In conclusion, we have shown here that the extra complexity of vortex patterns in asymmetrical mesoscopic superconductors, under a tilted field, can be used as a tool for vortex detection through the measurement of the transverse magnetization and the torque.

A.R.d.C.R. acknowledges the Brazilian agency CNPq for financial support. M.M.D. thanks CNPq, FAPERJ, Instituto do Milênio de Nanotecnologia (Brazil), and BOF/UA (Belgium). F.M.P. acknowledges support from the Flemish Science Foundation (FWO-Vl), the Belgian Science Policy (IUAP), and the ESF-AQDJJ network. 
*ton@if.ufrj.br

${ }^{1}$ A. T. Dorsey, Nature (London) 408, 783 (2000).

${ }^{2}$ B. J. Baelus, F. M. Peeters, and V. A. Schweigert, Phys. Rev. B 63, 144517 (2001).

${ }^{3}$ P. S. Deo, V. A. Schweigert, and F. M. Peeters, Phys. Rev. B 59, 6039 (1999).

${ }^{4}$ A. K. Geim, I. V. Grigorieva, S. V. Dubonos, J. G. S. Lok, J. C. Maan, A. E. Filippov, and F. M. Peeters, Nature (London) 390, 259 (1997).

${ }^{5}$ C. A. Bolle, V. Aksyuk, F. Pardo, P. L. Gammel, E. Zeldov, E. Bucher, R. Boie, D. J. Bishop, and D. R. Nelson, Nature (London) 399, 43 (1999).

${ }^{6}$ A. K. Geim, S. V. Dubonos, J. J. Palacios, I. V. Grigorieva, M. Henini, and J. J. Schermer, Phys. Rev. Lett. 85, 1528 (2000).

${ }^{7}$ I. V. Grigorieva, W. Escoffier, J. Richardson, L. Y. Vinnikov, S. Dubonos, and V. Oboznov, Phys. Rev. Lett. 96, 077005 (2006).

${ }^{8}$ A. Kanda, B. J. Baelus, F. M. Peeters, K. Kadowaki, and Y. Ootuka, Phys. Rev. Lett. 93, 257002 (2004).

${ }^{9}$ A. R. Jurelo, J. V. Kunzler, J. Schaf, P. Pureur, and J. Rosenblatt, Phys. Rev. B 56, 14815 (1997).

${ }^{10}$ C. Howald, P. Fournier, and A. Kapitulnik, Phys. Rev. B 64, 100504(R) (2001).

${ }^{11}$ T. Ekinoa et al., Physica C 426-431, 230 (2005).

${ }^{12}$ M. M. Doria and I. G. de Oliveira, Phys. Rev. B 49, 6205 (1994).

${ }^{13}$ V. G. Kogan, Phys. Rev. Lett. 89, 237005 (2002).

${ }^{14}$ Y. Wang, L. Li, M. J. Naughton, G. D. Gu, S. Uchida, and N. P.
Ong, Phys. Rev. Lett. 95, 247002 (2005).

${ }^{15}$ D. E. Farrell, C. M. Williams, S. A. Wolf, N. P. Bansal, and V. G. Kogan, Phys. Rev. Lett. 61, 2805 (1988).

${ }^{16}$ H. Won and K. Maki, Europhys. Lett. 34, 453 (1996).

${ }^{17}$ M. M. Doria, A. R. de C. Romaguera, and F. M. Peeters, Phys. Rev. B 75, 064505 (2007).

${ }^{18}$ P. S. Deo, V. A. Schweigert, F. M. Peeters, and A. K. Geim, Phys. Rev. Lett. 79, 4653 (1997).

${ }^{19}$ A. R. de C. Romaguera, M. M. Doria, and F. M. Peeters, Phys. Rev. B 75, 184525 (2007).

${ }^{20}$ E. H. Brandt, J. Low Temp. Phys. 42, 557 (1981).

${ }^{21}$ B. J. Baelus, D. Sun, and F. M. Peeters, Phys. Rev. B 75, 174523 (2007).

${ }^{22}$ M. M. Doria and A. R. de C. Romaguera, Europhys. Lett. 67, 446 (2004).

${ }^{23}$ J. A. Osborn, Phys. Rev. 67, 351 (1945).

${ }^{24}$ M. Beleggia, M. D. Graef, and Y. T. Millev, J. Phys. D 39, 891 (2006).

${ }^{25}$ V. A. Schweigert, F. M. Peeters, and P. S. Deo, Phys. Rev. Lett. 81, 2783 (1998).

${ }^{26}$ V. A. Schweigert and F. M. Peeters, Phys. Rev. Lett. 83, 2409 (1999).

${ }^{27}$ B. J. Baelus and F. M. Peeters, Phys. Rev. B 65, 104515 (2002).

${ }^{28}$ J. J. Palacios, Phys. Rev. Lett. 84, 1796 (2000).

${ }^{29}$ A. K. Geim, S. V. Dubonos, J. G. S. Lok, M. Henini, and J. C. Maan, Nature (London) 396, 144 (1998). 